\title{
FRÜHBRONZEZEITLICHE BEFESTIGUNGEN IM OBEREN ŽITAVATAL: PROZESSE VON SYNOIKISMOS UND DIOIKISMOS
}

\author{
N ILS M ÜLLER-SCHEEßEL - PHILIPP GERBECKS - \\ K N U T R A S M A N N
}

\begin{abstract}
Early Bronze Age Fortifications in the Upper Žitava Valley: Processes of Synoikismos and Dioikismos. During recent research in the Upper Žitava valley, previously unknown Early Bronze Age fortifications were discovered near Hul and Vlkas. They probably represented the seat of relatively small social groups. In the Žitava valley only the site Vráble-Fidvár grew beyond this early stage into a large settlement of an area of up to 12 ha. In the course of a process of synoicism, the other sites were abandoned voluntarily or involuntarily, and the social groups there probably moved to Vráble. We may assume that this has also led to social division and hierarchisation, with the newcomers being dominated by the small group that had initially occupied Vráble. At the end of the Early Bronze Age in the course of a kind of dioicism, the large settlement of Vráble was abandoned. Afterwards, small settlements again dominated the settlement pattern.
\end{abstract}

Keywords: Slovakia, Early Bronze Age, fortification, settlement development, geophysical prospection.

\section{EINLEITUNG}

Nach den Einträgen von ZENON (zenon.dainst. org) hat Jozef Bátora nicht weniger als zehn Beiträge zu frühbronzezeitlichen Befestigungen publiziert. Zuletzt hat er in seinem Werk zur Frühbronzezeit in der Slowakei zu diesem Thema ausführlich Stellung bezogen (Bátora 2018, 229-242). Eine besondere Stellung nimmt dabei die Anlage Fidvár bei Vráble in der Südwestslowakei ein (Abb. 1), die er gemeinsam mit der Römisch-Germanischen Kommission seit 2007 erforscht. ${ }^{1}$ Bei den Grabungen dort wurden Häuser, Gruben, das zugehörige Gräberfeld sowie das Umland erkundet. Von überragender Bedeutung sollte sich der Umstand erweisen, dass durch großflächige geophysikalische Prospektionen nicht nur der Kernbereich der Siedlung mit den Gräben, sondern auch das gesamte Umfeld erfasst werden konnte. Wie in einem Palimpsest zeigen sich die bronzezeitlichen Besiedlungsspuren, aber auch ältere (neolithische Siedlungen) und jüngere (römische Lagergräben) archäologische Strukturen. Die herausragenden Ergebnisse in Hinsicht auf die neolithischen Besiedlungsspuren waren die Initialzündung für ein weiteres durch die Deutsche Forschungsgemeinschaft gefördertes Projekt, ${ }^{2}$ dessen Feldforschungen ebenfalls zu den hier präsentierten Erkenntnissen beitrugen.

Da von vornherein die Erforschung der frühbronzezeitlichen Siedlung von Vráble-Fidvár nicht auf die Siedlung selbst beschränkt sein sollte, wurden auch umfangreiche Prospektionen im Oberen Žitavatal durchgeführt (Müller-Scheeßel u. a. 2016). Diese erlauben es, das theoretisch formulierte Konzept zur Entstehung und Entwicklung von Vráble-Fidvár und seinem Umland (Bátora $u$. a. 2012, 125 f.) mit empirischen Daten abzusichern. Mit der folgenden Darstellung verbinden wir die Hoffnung, dass der Jubilar noch viele Jahre dazu beitragen möge, die Forschung zur frühbronzezeitlichen Siedlungsentwicklung im Oberen Žitavatal entscheidend zu bereichern (Abb. 2).

\section{SYNOIKISMOS UND DIOIKISMOS}

Die archäologische Forschung der zurückliegenden Jahre kennzeichnet eine Erweiterung ihres methodischen Inventars wie auch ihrer Perspektive auf die archäologischen Fundplätze. Die Berücksichtigung der umliegenden Landschaft ist dabei zentral, hat aber methodische Vorläufer wie Arbeiten von K. Flannery (1976), A. Sherratt (1983) oder E. Lange, H. D. Knapp und L. Jeschke (1986), die erstaunlich weit zurückreichen. Die Siedlungsgrabung in Vráble war von Anbeginn eingebettet in eine landschaftsarchäologische Perspektive, die Auswahl des Platzes selbst war das Ergebnis ausgedehnter Oberflächenbegehungen in den Tallandschaften von Žitava und Gran seit 2002. Es

\footnotetext{
1 Seit 2011 mit Förderung der Deutschen Forschungsgemeinschaft im Projekt „Herausbildung und Niedergang des frühbronzezeitlichen Siedlungszentrums von Fidvár bei Vráble (Südwestslowakei). Untersuchungen zur Wirtschaft und Sozialstruktur und politischer Organisation eines Sozialverbandes und seines Umfeldes" (RA 557/5, 1.3).

2 SFB1266, Teilprojekt C2, Projektnummer 2901391021: „Die Dynamik von Siedlungskonzentration und Landnutzung in frühen sesshaften Gemeinschaften des Nordwestlichen Karpartenbeckens“, PI: M. Furholt. Siehe dazu z. B. Furholt u. a. 2020.
} 


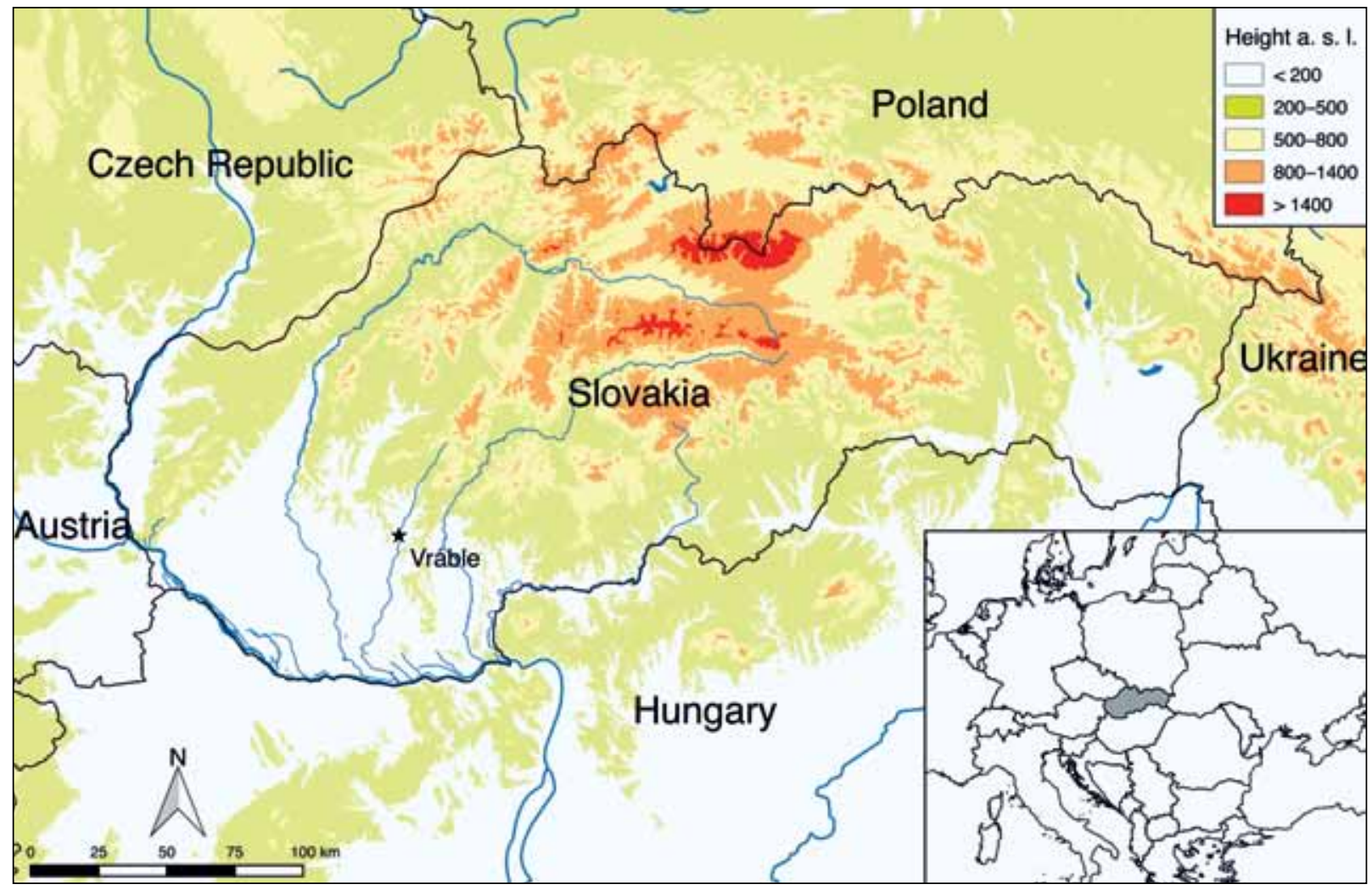

Abb. 1. Lokalisierung des Untersuchungsgebiets, symbolisiert durch den Fundplatz Vráble (Stern) in der Südwestslowakei (nach Rassmann u. a. 2017, 80, Abb. 1).

ist eine Landschaft, die J. Bátora, der in Nevidzany unweit von Vráble aufgewachsen ist, seit seinen Kindheitstagen wohlvertraut ist.

Die Notwendigkeit, die Geschichte einer Ansiedlung im Kontext der sie umgebenden Siedlungslandschaft zu untersuchen, zeigt sich am Beispiel von Fidvár bei Vráble überaus deutlich. Die Anlage der Siedlung, ihre schrittweise Erweiterung und der Niedergang ist mit der Wandlung der Siedlungslandschaft insgesamt verknüpft. Dazu gehören die Aufgabe von Siedlungen im Žitavatal, die offensichtliche Konzentration in der sich zu einer Großsiedlung entwickelnden Zentralsiedlung in der beginnenden sowie ihr Niedergang in der ausgehenden Frühbronzezeit, der wiederum zur Gründung von Siedlungen in der umliegenden Landschaft führt. Beide Prozesse - die Aufgabe von Siedlungen und in deren Folge die Agglomeration von Bevölkerungsgruppen an einem Ort, wie auch der entgegengesetzte Vorgang des Fortgangs von Bewohnern und die Gründung neuer Siedlungen - wurden umfassend unter den Begriffen Synoikismos für den Zusammenschluss und Dioikismos für das Auseinandergehen im Kontext der griechischen Polis erforscht (Pauly 1932). Die Gründe für die Bildung größerer politischer
Einheiten wie auch für ihren Zerfall waren dort vielfältig. Für unsere Betrachtung essentiell ist der Umstand, dass die Veränderungen, die Aufgabe von Siedlungen und deren Zusammenschluss, regelhaft durch ökonomische oder/und militärische oder/und politische Faktoren erzwungen wurden und den maßgeblichen Akteuren Raum für die Akkumulation von politischen und ökonomischen Einfluss eröffneten (Gebhardt 1977, 42).

\section{FIDVÁR BEI VRÁBLE}

Anhand der geophysikalischen Untersuchungen sowie gezielten Grabungen und Bohrungen ist es möglich, ein komplexes Bild der Entwicklung der Siedlung in Vráble-Fidvár zu zeichnen (Rassmann 2016; Skorna/Bátora/Kalmbach 2018). Die Siedlungsreste finden sich südlich der modernen Stadt Vráble auf einer Hochterrasse der Žitava (Bátora u. a. 2012).

Den Auftakt bildet etwa ab 2050 v. Chr. eine kleine 0,3-0,4 ha messende, grabenumwehrte Siedlung, deren Innenfläche durch Erosion der Žitava vollständig abgetragen wurde (Abb. 2). Erhalten sind spärliche Wallreste und Teile des ca. $4 \mathrm{~m}$ tiefen 


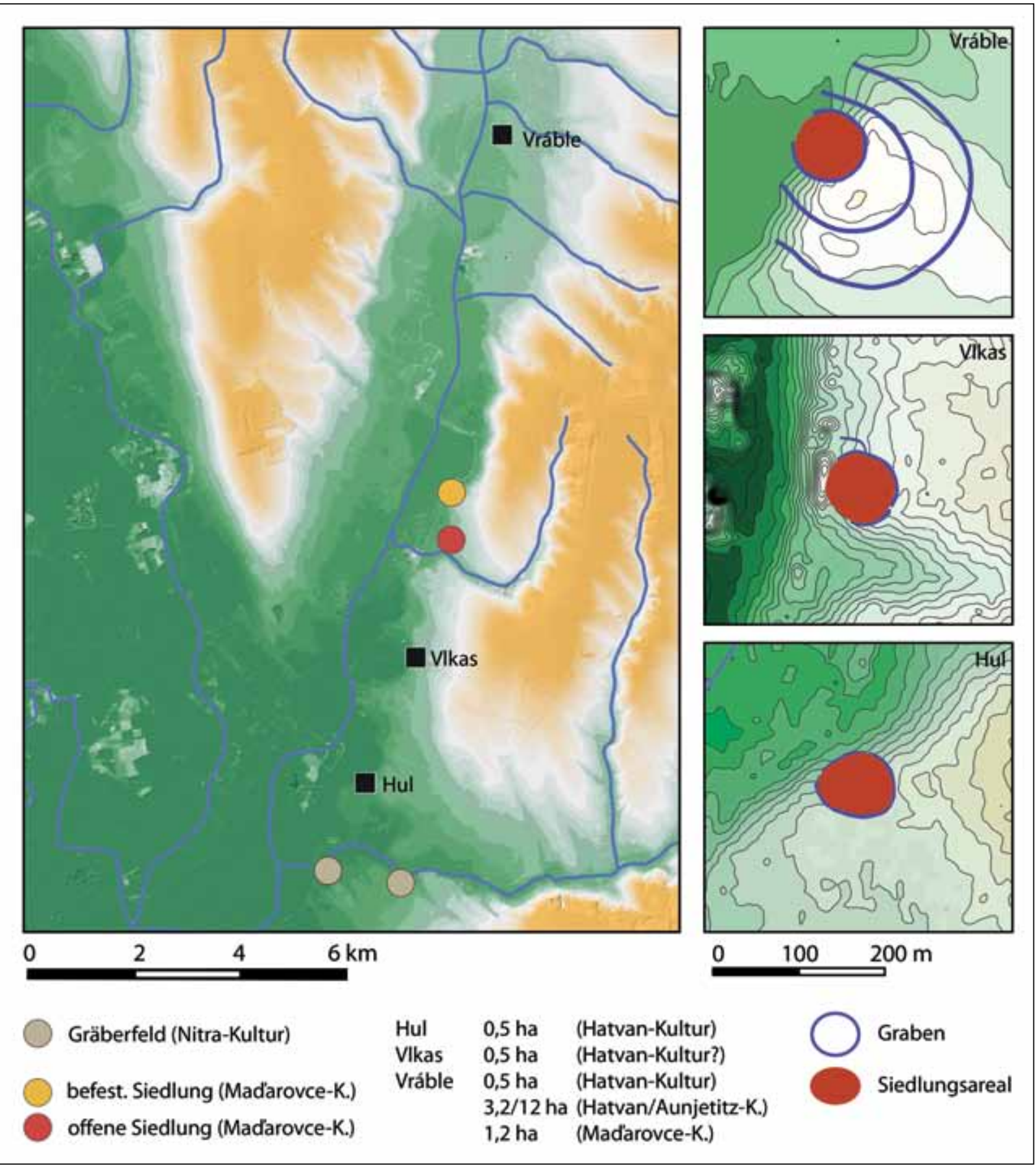

Abb. 2. Žitavatal mit den im Text erwähnten Fundplätzen Vráble, Vlkas und Hul. Weitere Fundplätze nach Bátora 2018, 76, 108, Abb. 69; 87 (Grafik by K. Rassmann).

Grabens (Behrens 2010; Nowaczinski u. a. 2015; Točík 1986). Die Siedlungsgröße entspricht der einer Reihe bisher bekannter frühbronzezeitlicher Siedlungen in der Slowakei wie auch der gemeinsam mit J. Bátora untersuchten Anlage in Rybnik (Bátoral Tóth 2014) wie auch jener der im Rahmen unserer gemeinsamen Untersuchungen entdeckten Anlagen von Hul und Vlkas (s. u.).
Etwa ab 1950 v. Chr. vergrößert sich die Siedlung in Vráble, und es wird eine aus Wall und Palisade bestehende Befestigungsanlage angelegt, die eine Innenfläche von ca. 3,6 ha mit ca. sechs Hausgruppen umschließt. In deren Peripherie finden sich weitere ca. acht Hausgruppen, wie nach den magnetischen Daten und Oberflächenaufsammlungen geschätzt werden kann. Ausgrabungen in zwei 


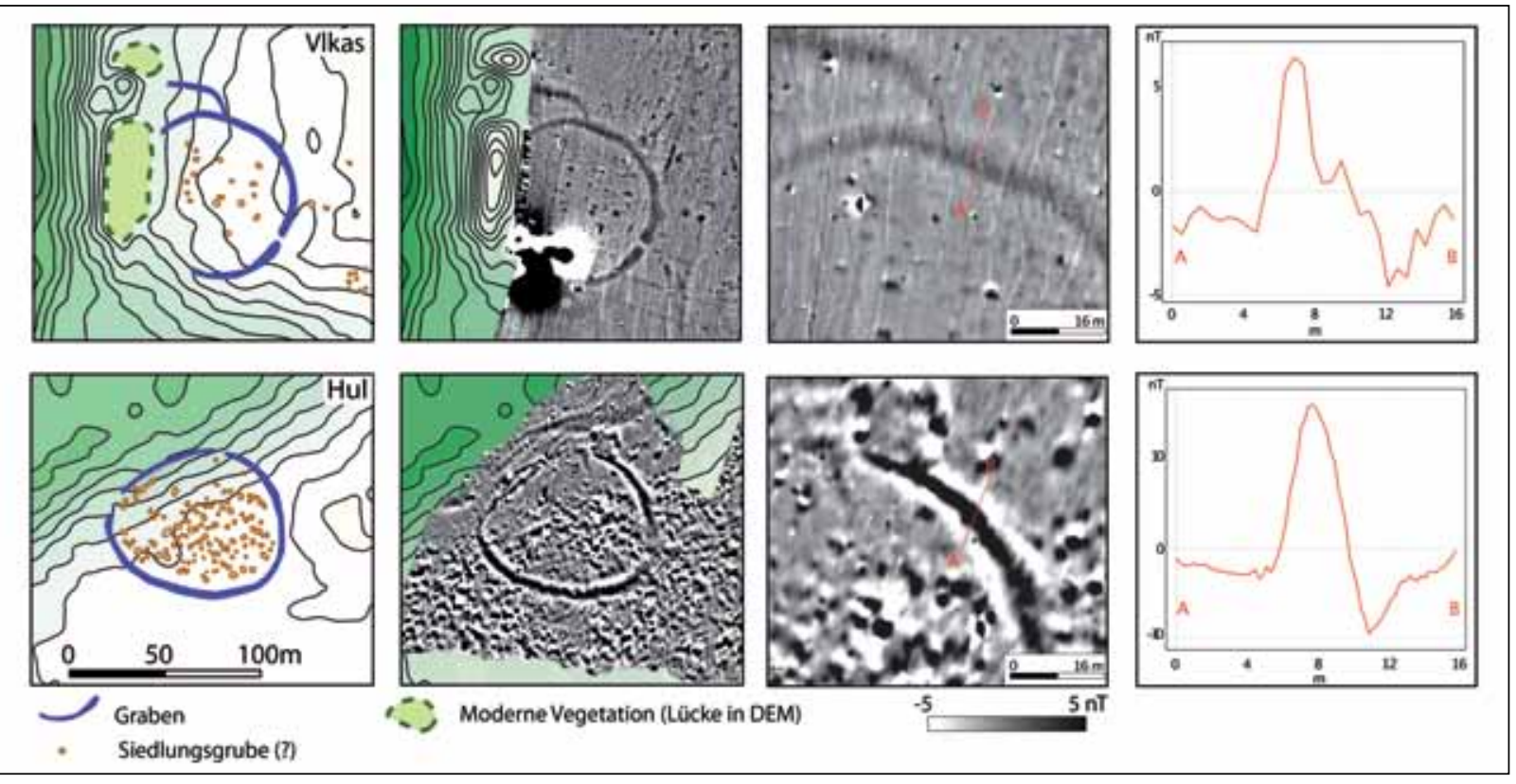

Abb. 3. Hul und Vlkas. Magnetikplan und dessen Umzeichnung sowie jeweils ein Profil durch den Graben (Graphik K. Rassmann).

Bereichen der Siedlung zeigen, dass um 1730 v. Chr. die Siedlung durch eine Brandkatastrophe zerstört wurde (Skorna/Bátora/Kalmbach 2018).

Das südlich der Siedlung gelegene Gräberfeld ist ausschnittweise untersucht (Stucky 2018). Nahezu alle Gräber sind durch Schächte gestört (Müller-Scheeßel u. a. 2020). Die Befundsituation spricht für eine gezielte Zerstörung und möglicherweise ritualisierte Entnahme von Beigaben und menschlichen Überresten. Die ${ }^{14} \mathrm{C}$-Daten platzieren einige der Aktivitäten in das Zeitfenster der Zerstörung der Siedlung um 1730 v. Chr.

Nach etwa 1730 v. Chr. erfolgt eine Verkleinerung der Siedlung. Eine aufwändig errichtete Befestigungsanlage aus Wall und 7-8 $\mathrm{m}$ breiten Graben umschließt nun eine Innenfläche von 1,2 ha, die Raum für maximal drei Hausgruppen bot. Die Siedlung bestand etwa bis 1600 v. Chr. und wird dann aufgegeben.

Das Anwachsen der Siedlung ist schwerlich durch ein Bevölkerungswachstum zu erklären, wahrscheinlicher wäre ein Zusammenschluss mehrerer Siedlungen (Synoikismos). Genauso wirft auch der Niedergang der Siedlung nach 1730 v. Chr. die Frage auf, ob die Mehrzahl der Bewohner der Zerstörung der Siedlung zum Opfer fiel oder aber die Siedlung verließ (Dioikismos). Die Beantwortung dieser Frage erfordert den Blick in die umliegende Landschaft. Darauf zielten mehrere
Prospektionskampagnen der zurückliegenden Jahre, wobei sich die Arbeiten der Frankfurter und Kieler Arbeitsgruppen ergänzten (Breitenfeld u. a., in Vorbereitung; Müller-Scheeßel u. a. 2016).

\section{HUL}

Der Fundplatz nördlich der heutigen Ortschaft Hul auf der Flur Kratiny war bereits vor den Prospektionen des Vráble-Projekts als mehrperiodiger Siedlungsplatz bekannt (Samuel 1995; zu den nachfolgenden Ergebnissen siehe teilweise Müller-Scheeßel u. a. 2016, 85-89). Er liegt ungefähr $12 \mathrm{~km}$ südlich von Vráble-Fidvár hochwassergeschützt auf der linken Terrasse der Žitava. Bei einer magnetischen Prospektion wurde 2014 ein Kreisgraben von ungefähr $80 \mathrm{~m}$ Durchmesser nachgewiesen, in dessen Bereich bei systematischen Begehungen eindeutig frühbronzeitliches Fundmaterial aufgesammelt wurde. Weitere geoarchäologische Untersuchungen wurden von der Arbeitsgruppe Heidelberg in den Folgejahren vorgenommen.

Der vollständig erfasste Kreisgraben ist von leicht ellipsoider Form und misst in west-östlicher Richtung ungefähr $90 \mathrm{~m}$ und in nord-südlicher $72 \mathrm{~m}$. Damit umschließt er eine Fläche von 0,5 ha. Der Graben hat in den am höchsten gelegenen Bereichen eine Breite von bis zu $5 \mathrm{~m}$, hangabwärts

\footnotetext{
3 Die Heidelberger Arbeiten mündeten 2016 in die unveröffentlichte Masterarbeit „Geoarchäologische Untersuchung der frühbronzezeitlichen Siedlung bei Hul, Slowakei“ von Philipp Gerbecks.
} 
ist er deutlich schmaler. Dies korrespondiert mit sehr unterschiedlichen Tiefen, wie zahlreiche Bohrungen der Frankfurter und Heidelberger Arbeitsgruppen gezeigt haben. An den breitesten Stellen erreicht der Graben auch seine größte Tiefe von bis zu 4,4 m. Die unterschiedliche Breite und Tiefe ist also in erster Linie eine Folge der Erosion. Ein eindeutiger Eingang in Form etwa einer Erdbrücke war nicht festzustellen. Zwar sind oberhalb der Hang- und Erosionskante, die quer durch die Kreisgrabenanlage verläuft, zahlreiche Anomalien festzustellen, doch sind diese von unspezifischer Form und lassen sich deshalb chronologisch nicht weiter eingrenzen.

Neben einer schwarzpolierten frühbronzezeitlichen Scherbe in einem der Bohrprofile (Müller-Scheeßel u. a. 2016, 86) zeigen auch zwei ${ }^{14}$ C-Proben ein eindeutig frühbronzeitliches Alter der Grabenanlage an (Gerbecks 2016). Jeweils ein ${ }^{14} \mathrm{C}$-Datum stammt aus zwei Bohrungen in den Graben, die von Proben dicht über der Grabensohle bei $367 \mathrm{~cm}$ (HUL1) bzw. $431 \mathrm{~cm}$ (HUL2) unter der heutigen Oberfläche entnommen wurden. Bei der Evaluierung der Proben ist allerdings zu berücksichtigen, dass es sich dabei um Holzkohle handelt, bei der keine Aussage zur Langlebigkeit des Probenmaterials getroffen werden kann. Ein "Altholzeffekt" kann also grundsätzlich nicht ausgeschlossen werden. Die Probe aus der Bohrung HUL1 (MAMS-24483) ergab ein unkalibriertes Alter von 3710 +/- 24 BP, dasjenige von HUL2 (MAMS-24485) ein Alter von 3460 +/- 30. Kalibriert errechnet OxCal (vers. 4.3) daraus im 2-sigma-Bereich (68,2 \%) ein Alter von 2140-2039 calBC (HUL1) bzw. 1874-1698 calBC (HUL2). Sofern hier nicht ein Altholzeffekt vorliegt (s. o.), zeigen diese Ergebnisse an, dass die Besiedlung in Hul mit dem Beginn der Frühbronzezeit einsetzte und mindestens 150 Jahre andauerte. Da die Sedimentanalysen eher auf ein allgemeines Zusedimentieren des Grabens hindeuten (Müller-Scheeßel u. a. 2016, 89), stellen die ${ }^{14} \mathrm{C}$-Daten allerdings nur grobe termini ante quem dar.

Anders als in Vráble-Fidvár gibt es im magnetischen Bild keine sicheren Anzeichen von Häusern, weder innerhalb des Grabens noch außerhalb. Zwar ist zu berücksichtigen, dass potentiell zahlreiche Strukturen anderer Perioden - von der Fundstelle liegen außer frühbronzezeitlichen Funden auch solche der Bandkeramik, der Lengyel- und der Badener Kultur, der Latène- und der römischen Zeit und aus dem Mittelalter vor (Samuel 1995) - das Bild verunklaren, aber ähnliche Überlagerungen gibt es auch in Vráble. Insofern ist davon auszugehen, dass entweder keine vergleichbaren Strukturen wie in Vráble existierten oder dass diese Strukturen nicht in derselben Weise wie in Vráble konserviert wurden, d. h. nicht abgebrannt sind. Trotz dieser Unsicherheiten sollte man unseres Erachtens derzeit davon ausgehen, dass sich die frühbronzezeitliche Besiedlung auf das Innere der Grabenanlage beschränkte. Mit einer Fläche von 0,5 ha hätte es allenfalls Platz für eine Hausgruppe geboten. Interessanterweise entspricht dies ungefähr der Größe der Hauscluster, die in Vráble-Fidvár identifiziert worden sind (s. o.).

Zusammenfassend lässt sich sagen, dass wir in Hul vermutlich eine kleine Siedlung der Frühbronzezeit von ungefähr 50-80 Bewohnern erfassen, die sich mit einem angesichts der geringen Größe bemerkenswert mächtigen Graben umgeben haben. Laut den verfügbaren ${ }^{14} \mathrm{C}$-Daten wurde sie im Laufe der Frühbronzezeit verlassen. Von Hul sind ferner zwei Grabfunde der Nitra-Kultur bekannt (Abb. 2), jedoch ist angesichts der Entfernung von ungefähr $2 \mathrm{~km}$ sehr unsicher, ob sie den Bewohnern der Befestigung zuzuweisen sind.

\section{VLKAS}

Ungefähr $2 \mathrm{~km}$ nördlich von Hul, d. h. $10 \mathrm{~km}$ südlich von Vráble-Fidvár wurde bei großflächigen magnetischen Prospektionen zwischen den modernen Dörfern Maňa und Vlkas ${ }^{4}$ auf den Fluren Baroč und Za hlbokou cestou ein bisher unbekanntes Grabenwerk entdeckt (Abb. 2; 4). Insgesamt wurde auf einer linksseitigen hochwassergeschützten Terrasse der Žitava eine Fläche von 27 ha gemessen. Zwar verläuft die Žitava modern mehr als $1 \mathrm{~km}$ weiter westlich, im Luftbild ist jedoch ein alter Flusslauf nur wenige 100 m entfernt zu erkennen. Neben der hier diskutierten Kreisgrabenanlage wurde im Norden der Fläche eine bereits bekannte bandkeramische Siedlung (Točík 1981, 301) - das eigentliche Ziel der damaligen geophysikalischen Untersuchungen sowie Befunde weiterer Zeitstufen festgestellt.

Die wohl prägnanteste Anomalie ist die Kreisgrabenanlage am westlichen Rand des prospektierten Bereichs bei 298100/5334850 (WGS84, UTM 34N). Tatsächlich ist die kreisrunde Anlage auch auf Luftbildern zu erkennen (z. B. GoogleEarth, 29. 06. 2016 oder 27. 03. 2019; Abb. 5).

Dem magnetischen Bild zufolge hat die Anlage einen Durchmesser von ungefähr $80 \mathrm{~m}$, was bei einem Vollkreis einer Fläche von 0,5 ha entsprechen würde. Davon sind allerdings nur 0,4 ha erhalten

\footnotetext{
4 Die Kreisgrabenanlage berührt die moderne Gemarkungsgrenze zwischen den Dörfern Maňa und Vlkas, sie liegt aber größtenteils auf der Gemarkung Vlkas.
} 


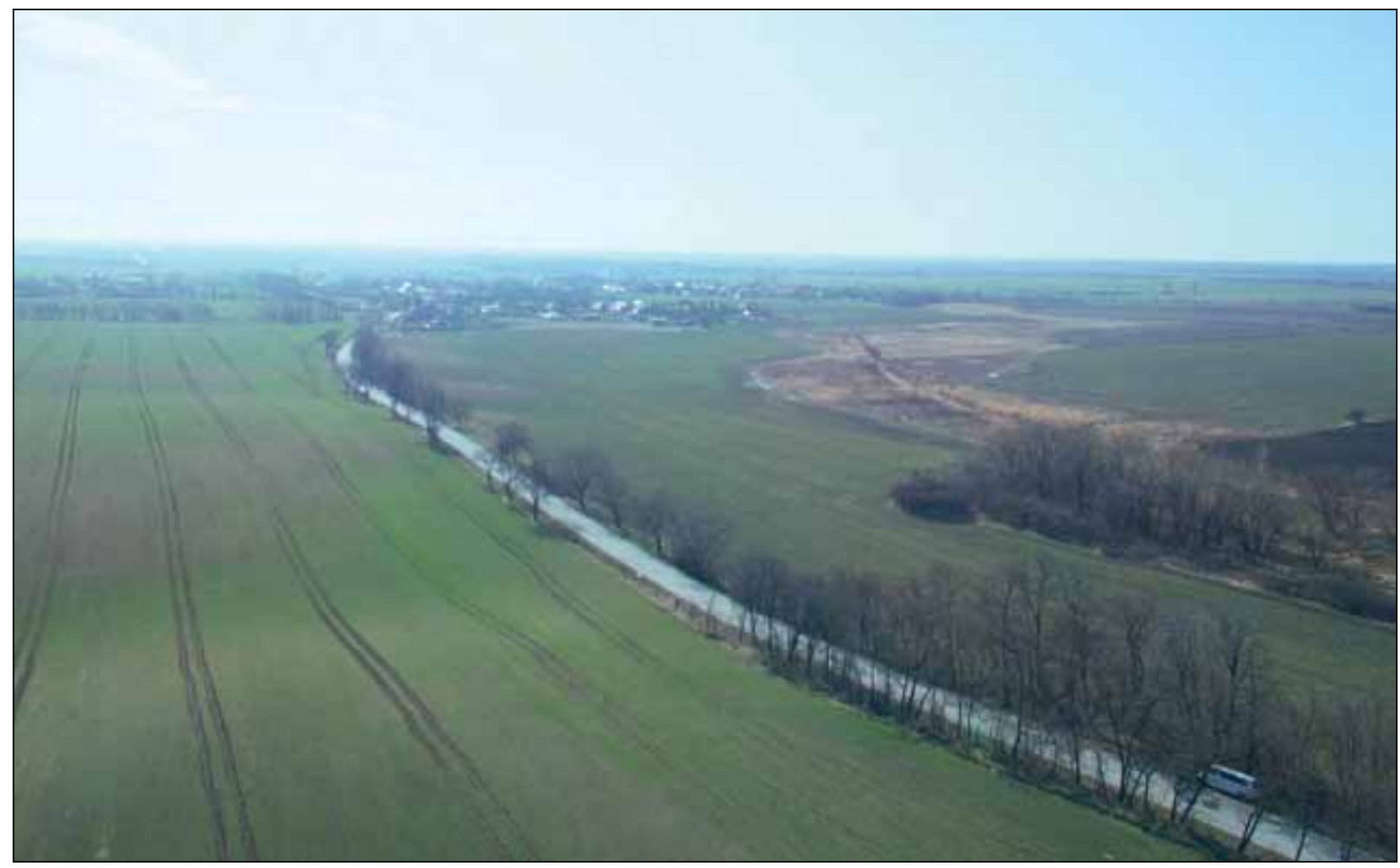

Abb. 4. Vlkas. Blick nach Süden. Die Kreisgrabenanlage liegt direkt an der Straße in der unteren Bildmitte (Foto N. Müller-Scheeßel).

bzw. erkennbar. Das westliche Ende ragte vermutlich über den heutigen Hang hinaus, ist also entweder durch die Erosion des Hanges oder bei Anlage der modernen Straßenführung zerstört worden. Ferner ist der südliche Bereich durch eine moderne, stark ausstrahlende Anomalie in großen Teilen maskiert.

Die nahezu kreisrunde Anomalie ist auch in Analogie zur Anlage von Hul zwanglos als Graben anzusprechen. Sie weist Werte bis zu $4 \mathrm{nT}$ auf. Der östliche Teil scheint eine Breite von bis zu $6 \mathrm{~m} \mathrm{zu}$ besitzen, nach Westen hin verjüngt sich der Graben deutlich: Im Nordwesten beträgt die Breite lediglich noch $3 \mathrm{~m}$. Da das Gelände zur Žitava hin leicht abfällt, ist auch hier wie für Hul zu überlegen, ob nicht eine unterschiedlich starke Erosion zu den abweichenden Werten geführt hat. Demnach wäre der Graben im Osten am tiefsten, während die westlich gelegenen Abschnitte möglicherweise nur noch wenige Dezimeter tief erhalten sind. Nicht auszuschließen ist allerdings auch, dass es sich bei den unterschiedlichen Grabenbreiten um eine absichtliche Maßnahme handelt. Ausgerechnet an der breitesten Stelle befindet sich nämlich der einzige sichtbare Durchgang durch den Graben. Dieser ist offensichtlich als Erdbrücke von $6 \mathrm{~m}$ Länge und $3 \mathrm{~m}$ Breite ausgebildet. Ein Wall ist im Magnetogramm nicht erkennbar, ein solcher zeichnet sich auch im mittels Structure-from-Motion erzeugten feinmaschigen Höhenmodell nicht ab (nicht abgebildet). Entweder wurde der Aushub des Grabens also nicht zu einem Wall aufgeschüttet oder ein ehemals vorhandener Wall ist komplett erodiert.

Zwei schmale west-östliche verlaufende Anomalien befinden sich im Süden der Anlage. Schnurgerade und perfekt parallel sind sie im Magnetikbild über ca. $100 \mathrm{~m}$ zu verfolgen, im Luftbild sogar über $200 \mathrm{~m}$. Da die nördliche der beiden Linien den Graben überschneidet, sind sie eindeutig jünger als dieser. Weniger eindeutig ist die Deutung bei einer Ausbuchtung an der östlichsten Stelle des Grabens. $\mathrm{Ob}$ es sich um ein in funktionalem Zusammenhang mit dem Graben stehendes Objekt handelt oder eine Grube anderer Zeitstellung, die zufällig mit dem Graben verschmolzen ist, muss derzeit offen bleiben. Ferner gibt es nördlich der Anlage noch zwei deutliche längliche Anomalien, die bis an die westliche Kante des Magnetikbildes verlaufen. Das östliche Ende der südlicheren der beiden biegt nach Süden um und verschwindet scheinbar im Graben. Zumindest bei dieser scheint ein funktionaler Zusammenhang mit der Anlage denkbar, auch wenn nicht klar ist, worin dieser bestanden haben könnte. Schließlich sind innerhalb und außerhalb des Grabens zahlreiche Anomalien 


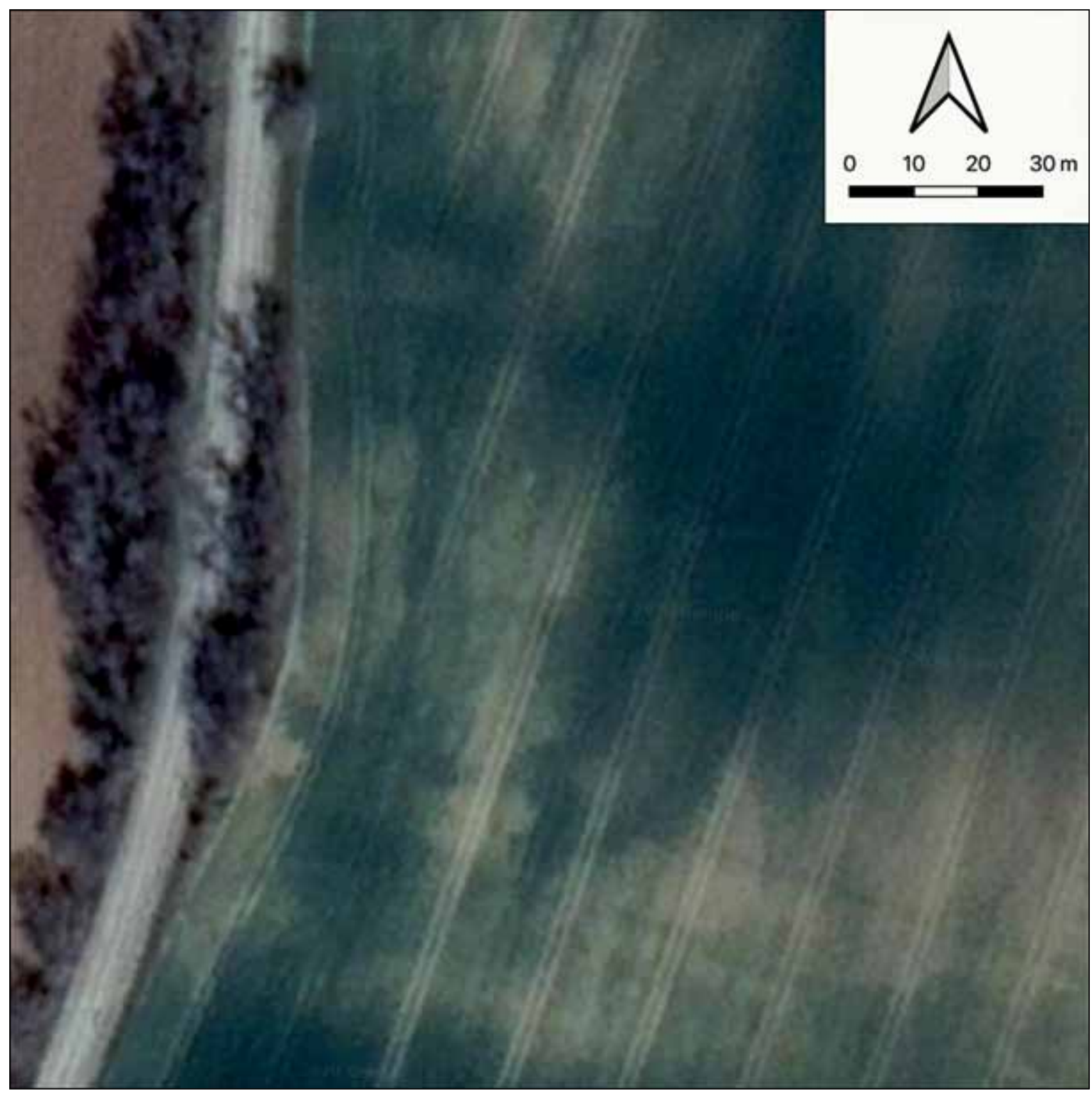

Abb. 5. Vlkas. Luftbild der Kreisgrabenanlage (Foto GoogleEarth, 27. 03. 2019).

unklarer Form zu erkennen. Außerhalb des Grabens finden sie sich vor allem nordöstlich und nördlich der Anlage. Bei einigen der länglichen Strukturen könnte man an bandkeramische Längsgruben denken, aber so eindeutig wie am nördlichen Rand der prospektierten Fläche ist die Ansprache in diesem Fall nicht. Vor allem innerhalb des Grabens sind rundliche Anomalien zu finden, die in der Mehrzahl Gruben repräsentieren dürften. Abgesehen von der räumlichen Nähe dieser potentiellen Gruben und des Grabens gibt es allerdings derzeit keine Anhaltspunkte, die Aufschluss über die chronologische Beziehung geben würden. Eine eindeutige Innenbebauung der Anlage etwa in der Form von Häusern lässt sich nicht feststellen.
Die Übereinstimmungen zwischen den Anlagen von Hul und Vlkas sind bemerkenswert. Angefangen bei der Größe über die Lage an der Terrassenkante bis zur scheinbar fehlenden Innenbebauung scheinen sie mehr oder weniger exakte Zwillinge. Auch wenn datierbares Fundmaterial bisher fehlt, kann man unseres Erachtens deshalb davon ausgehen, dass die Anlage von Vlkas ebenfalls frühbronzezeitlich datiert.

\section{WEITERE ANLAGEN?}

Angesichts der Ähnlichkeiten zwischen den Anlagen von Hul und Vlkas sowie dem rekonstruierten Frühzustand von Vráble-Fidvár ist es 
verlockend, nach weiteren ähnlichen Befestigungen Ausschau zu halten. Leider ist damit zu rechnen, dass etwaige derartige Fundstellen zumindest teilweise von den heutigen Ortskernen, die ebenfalls stets an den Terrassenkanten der Žitava liegen, zerstört wurden. Potentielle Anlagen sind also nur zwischen den heutigen Ortschaften zu entdecken.

Tatsächlich existieren nördlich von Maňa gleich zwei auffällige Anomalien in Luftbildern (z. B. GoogleEarth, 08. 06. 2017), und bei einer $-3,2 \mathrm{~km}$ nördlich der Anlage von Vlkas - wird in der Literatur eine befestigte Siedlung der Madarovce-Kultur angenommen (Abb. 2). Leider gelang es dort jedoch wegen ungünstiger Bepflanzungszyklen noch nicht, geophysikalische Untersuchungen durchzuführen, um die Verdachtsmomente zu erhärten.

\section{DISKUSSION - SYNOIKISMOS VS. DIOIKISMOS}

Bereits 2012 wurde die Entwicklung in Vráble-Fidvár in Zusammenhang mit der Siedlungsentwicklung im gesamten Žitavatal gebracht (Bátora u. a. 2012, 125 f.). Schon bei der Gelegenheit wurde darauf hingewiesen, dass aus der geringen Anzahl von bekannten Siedlungen in der Mitte der Frühbronzezeit und der Zunahme von Siedlungen der Madarovce-Kultur am Ausgang der Frühbronzezeit ein Zusammenhang mit dem Aufstieg und Niedergang der Siedlung von Fidvár bei Vráble bestand. Die Entdeckung von Hul und Vlkas ist aus mehreren Gründen bedeutsam. Zum einen entspricht ihre Zeitstellung der der Pioniersiedlung von Fidvár. Übereinstimmend ist auch die Größe der Siedlungen. Der dritte Aspekt ist ihre Befestigung durch einen tiefen Graben vergleichbarer Größe. Im Unterschied zu Fidvár bestanden die Siedlungen in Hul (sicher) und Vlkas (wahrscheinlich) jedoch nur kurze Zeit, vielleicht drei bis fünf Generationen. Können wir annehmen, dass die Bewohner diesen Platz aufgegeben und sich an anderer Stelle neu angesiedelt haben, vielleicht in Fidvár? Wie oben betont, ist das Anwachsen der Siedlung in Fidvár sicher nicht auf Bevölkerungswachstum zurückzuführen, sondern auf einen Zuzug von außen.

Bedeutsam ist der Umstand, dass der Friedhof südlich der Siedlung von Fidvár bei Vráble mit ca. 5 ha eine relativ geringe Größe besitzt. Diese weist darauf hin, dass hier wahrscheinlich nur eine Gemeinschaft von ca. 40-80 Bewohnern ihre Toten bestatteten. Wir schätzen nach den Ausgrabungen und den magnetischen Prospektionsdaten, hier sind die sekundären Graböffnungen gut zu erkennen, eine Obergrenze von 500-1000 Bestattungen
(Müller-Scheeßel u. a. 2020). Wäre der Bestattungsplatz zur Blütezeit der Siedlung von allen 12-14 Hausgruppen genutzt worden, wäre mit deutlich mehr Gräbern und einer größeren Ausdehnung des Friedhofes zu rechnen. Das wahrscheinlichste Szenario ist eine Nutzung durch die Sozialgruppe, von der die Siedlung gegründet wurde. Geht man von einer derartigen Annahme aus, stellt sich die Frage, wo die anderen Bewohner der Siedlung von Fidvár ihre Toten bestatteten. Zu vermuten ist eine Weiternutzung ihrer alten Bestattungsplätze.

Diese Konstellation scheint beispielsweise auf das Gräberfeld von Jelšovce zuzutreffen, in dessen Umfeld eine Siedlung liegt, die von uns vollständig magnetisch prospektiert wurde (unpubliziert). Die Nutzungsdauer dieser Siedlung ist aber mit Sicherheit kürzer als die Belegungsdauer des Gräberfeldes von etwa 2050-1600 v. Chr. (Bátora 2000). Folglich wurde hier der Siedlungsplatz aufgegeben und verlagert, der Bestattungsplatz jedoch beibehalten. Leider haben wir bei den bisherigen Prospektionen keine belastbaren Daten zu weiteren Gräberfeldern im Žitavatal gewinnen können.

Ebenso wie die angestammten Grabplätze vermutlich weitergenutzt wurden, scheint es denkbar, dass auch die Ackerfluren nicht komplett aufgegeben wurden. Das legt allein schon die Notwendigkeit nahe, in Vráble eine Bevölkerung von mehreren Hundert Menschen zu ernähren. Aber auch die in die Tausenden gehenden Vorratsgruben (Bátora u. $a$. 2012) bezeugen eine umfangreiche Vorratshaltung. Für die ehemaligen Bewohner von Vlkas und Hul hätte das aber selbstverständlich einen bedeutenden Mehraufwand und Nachteil bedeutet. Antwort auf diese Frage von möglicherweise unterschiedlichen Ackerflächen können eventuell Isotopenanalysen an Getreideresten liefern, deren Auswertung unmittelbar vor dem Abschluss steht.

Vlkas, Hul und die früheste Siedlung von Fidvár markieren eine Aufsiedlung des Žitavatals in der beginnenden Frühbronzezeit durch befestigte Siedlungen. Die regelhaft erscheinenden Abstände von 2-3 km könnten darauf hindeuten, dass dabei territoriale Abgrenzungen eine Rolle spielten. Die Aufgabe der Siedlungen von Vlkas und Hul, aber auch der Aufstieg der Siedlung von Vráble legt nahe, das die kleinen Befestigungsanlagen nicht ausreichend Schutz boten und als Alternative ein Zusammenschluss mehrerer Siedlungen erfolgte. Das Siedlungsbild in der mittleren Frühbronzezeit spiegelt diesen Prozess durch das Fehlen von Siedlungen im Umfeld von Fidvár. Erst in der ausgehenden Frühbronzezeit in der Mad’arovce-Kultur beobachten wir eine erneute Aufsiedlung der Landschaft, ein Vorgang, der mit der Verkleinerung von Fidvár einhergeht (Abb. 6). 


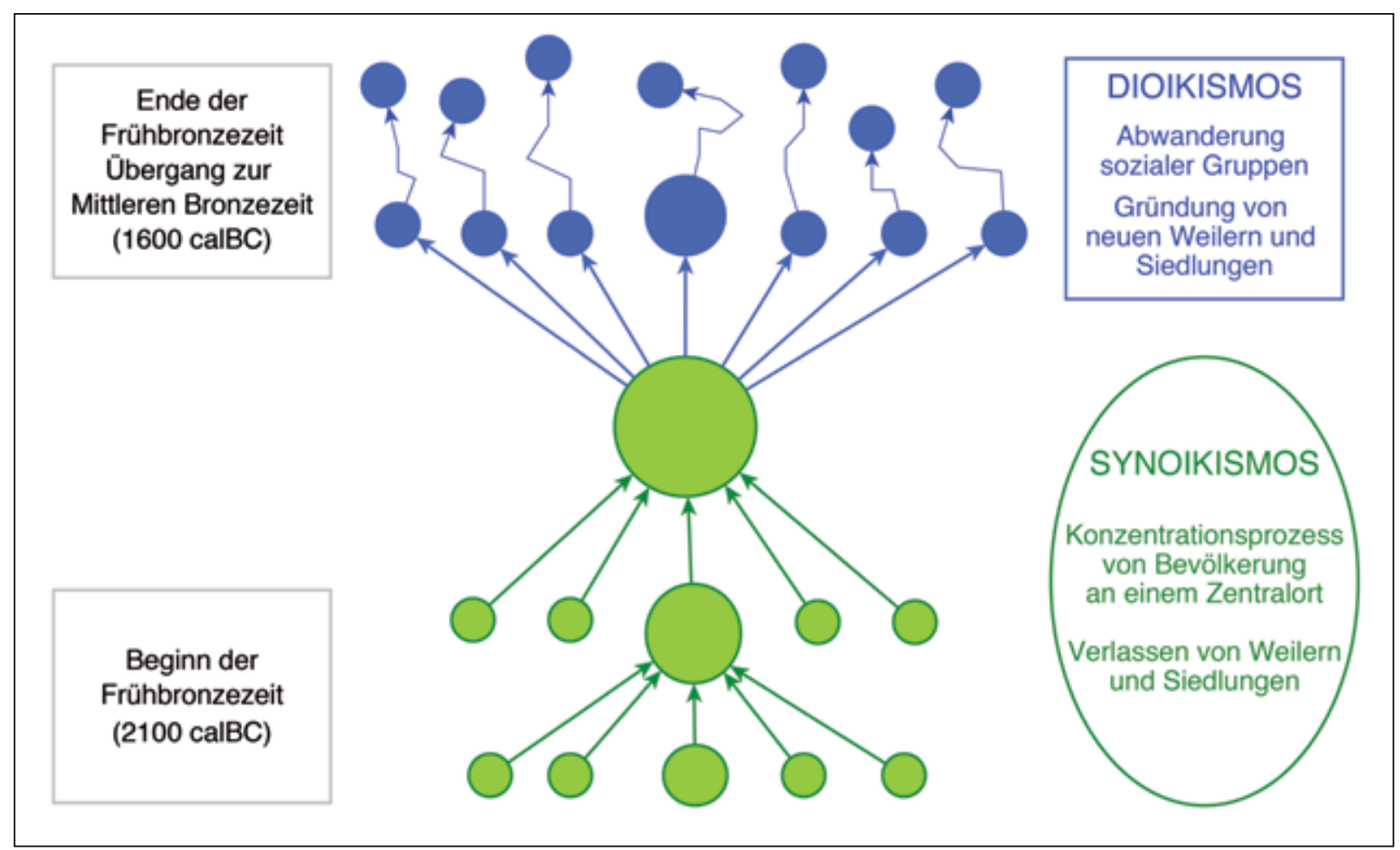

Abb. 6. Schema der Prozesse von Synoikismos und Dioikismos am Beispiel der Frühbronzezeit des Žitavatals (Grafik K. Rassmann).

Die Gründe für diese Veränderung liegen im Dunkeln. Die zentrale Rolle von Befestigungsanlagen im Siedlungsbild, die sekundären Graböffnungen wie auch die zahlreichen Traumata an den Skeletten des Gräberfeldes von Fidvár zeugen von Konflikten und den Notwendigkeiten der in Fidvár zusammen siedelnden Sozialgruppen, militärische Strategien zu entwickeln. Die Aufgabe der kleinen Befestigungsanlagen und die Zentralisation in einer großen Anlage scheint sich für ca. acht Generationen, d. h. ca. 200 Jahre bewährt zu haben und eine Heimstatt für etwa 800 Bewohner gewesen zu sein, ehe sie nach einer Zerstörung aufgegeben wurde. $\mathrm{Ob}$ hier innere oder äußere Konflikte ausschlaggebend waren, ist noch offen. Bei der Auswertung der sekundären Graböffnungen deuten sich mehrere Zeitfenster an, was sowohl als Anzeichen für innere wie auch äußere Spannungen gewertet werden könnte, zumal die Störung der Gräber durch die Schächte derart präzise erfolgte, dass "Insiderwissen“ vorausgesetzt werden muss.
Wir vermuten deshalb Akteure aus dem Umfeld der Siedlung.

Nach J. Burckhardt war der Synoikismos in der Frühzeit der griechischen Polis von Zwang beherrscht und forderte immer auch Opfer. ${ }^{5}$ Vielleicht waren äußerer Druck oder Zwang letztendlich die Auslöser für die Entstehung von Fidvár, zugleich waren sie aber auch der Keim des Niederganges. Ein konfliktgeladener Zusammenschluss vollzog sich am Beginn der Frühbronzezeit, für dessen Konfliktmanagement letztendlich noch nicht die entscheidenden sozialen Institutionen und Techniken zur Verfügung standen, um für mehr als 200 Jahre zu überdauern.

\section{SCHLUSS}

Die Südwestslowakei stellt eine archäologisch hervorragend erschlossene Region dar; dies gilt gerade auch und besonders für das Žitavatal. Vor

\footnotetext{
5 „Allein aus den Beispielen der hellen historischen Zeit lernt man auch die Opfer kennen, welche ein solcher Synoikismos kostete: gewaltsame Übersiedlung der Widerstrebenden oder auch ihre Zernichtung; was sich vollends nur ahnen läßt, ist der Jammer der Vielen, welche sich zwar fügten, aber ihre bisherigen Dörfer, Ortschaften und Städtchen verlassen mußten oder dieselben nur noch mit geringerer Sicherheit und vermindertem Wohlstand bebauen und bewohnen konnten. Schon allein die Entfernung von den Gräbern der Ahnen muß für den Griechen ein Unglück gewesen sein; er mußte nun den Totenkult verabsäumen oder er konnte denselben nur mit Schwierigkeiten vollziehen; jedenfalls hatte er das Ahnengrab nicht mehr täglich vor Augen“ (Burckhardt 1898-1902 (2014), 22 f.).
} 
diesem Hintergrund ist es ebenso überraschend wie erfreulich, dass dennoch immer wieder bisher unentdeckte Fundplätze das Siedlungsbild bereichern. Entscheidend tragen dazu Luftbilder bei, die heutzutage in völlig anderem Maße verfügbar sind als noch vor wenigen Jahrzehnten, aber auch neuartige großflächige geophysikalische Prospektionen.

$\mathrm{Zu}$ diesen Neuentdeckungen zählen auch die hier besprochenen sicher bis mutmaßlich frühbronzezeitlichen Grabenwerke von Hul und Vlkas, die vermutlich den Rückzugsort relativ kleiner Sozialverbände darstellten. Diese Familienverbände dürften auf demselben sozialen Niveau miteinander agiert haben. Im Žitavatal kommt nur der Fundplatz
Vráble-Fidvár über dieses Frühstadium hinaus und wächst zu einer Großsiedlung mit 12 ha Grundfläche. Im Zuge eines Synoikismosprozesses sind die anderen Fundplätze freiwillig oder unfreiwillig aufgegeben worden, und die dortigen Familien sind vermutlich nach Vráble umgesiedelt. Wir dürfen annehmen, dass es dabei auch zu einer sozialen Aufspaltung und Hierarchisierung gekommen ist, indem die Neuankömmlinge von der bereits vorher ansässigen Kleingruppe dominiert wurden. Am Ende der Frühbronzezeit kommt es dann zum Verlassen der Großsiedlung im Zuge eines Dioikismos. Danach dominieren wieder Kleinsiedlungen das Siedlungsbild.

\section{LITERATUR}

Bátora 2000 - J. Bátora: Das Gräberfeld von Jelšovce/Slowakei. Ein Beitrag zur Frühbronzezeit im nordwestlichen Karpatenbecken. Teil 1-2. PAS 16. Kiel 2000.

Bátora 2018 - J. Bátora: Slovensko v staršej dobe bronzovej. Bratislava 2018.

Bátora u. a. 2012 - J. Bátora/A. Behrens/J. Gresky/ M. Ivanova/K. Rassmann/P. Tóth/K. Winkelmann: The rise and decline of the Early Bronze Age settlement Fidvár near Vráble, Slovakia. In: J. Kneisel/W. Kirleis/ M. Dal Corso/N. Taylor/V. Tiedtke (eds.): Collapse or continuity? Environment and development of Bronze Age human landscapes. Proceedings of the International Workshop 'Socio-Environmental Dynamics over the last 12.000 years: The creation of landscapes $2\left(14^{\text {th }}-18^{\text {th }}\right.$ March 2011)' in Kiel. Bonn 2012, 111-129.

Bátora/Tóth 2014 - J. Bátora/P. Tóth: Settlement Strategies in the Early Bronze Age in South-Western Slovakia. In: T. L. Kienlin (ed.): Settlement, communication and exchange around the Western Carpathians. International Workshop held at the Institute of Archaeology, Jagiellonian University, Krakow, October 27-28, 2012. Oxford 2014, 325-340.

Behrens 2010 - A. Behrens: Fidvár bei Vráble. Untersuchungen zu der Befestigung A. Unpublizierte Diplomarbeit. Christian-Albrechts-Universität Kiel. Kiel 2010.

Breitenfeld u. a., in Vorbereitung - B. Breitenfeld/N. MüllerScheeßel/I. Cheben/M. Furholt: Results of non-invasive prospection methods in LBK and Želiezovce settlement sites of the Upper Žitava Valley. In: M. Furholt/ I. Cheben/A. Bistáková/M. Wunderlich (eds.): Archaeology in the Žitava Valley II. The LBK and Želiezovce settlement sites of Vlkas and Úlany. Amsterdam, in Vorbereitung.

Burckhardt 1898-1902 (2014) - J. Burckhardt: Griechische Kulturgeschichte I-IV. Berlin 1898-1902 (2014).

Flannery 1976 - K. V. Flannery: The Early Mesoamerican village. Studies in archeology. New York 1976.

Furholt u. a. 2020 - M. Furholt/N. Müller-Scheeßel/ M. Wunderlich/I. Cheben/J. Müller: Communality and Discord in an Early Neolithic Settlement Agglomeration: The LBK Site of Vráble, Southwest Slovakia. Cambridge Archaeological Journal 30, 2020, 469-489.

Gebhardt 1977 - J. Gebhardt: Strukturprobleme einer Revolutionstheorie. Zeitschrift für Politik 24, 1977, 132-455.
Gerbecks 2016 - P. Gerbecks: Geoarchäologische Untersuchung der frühbronzezeitlichen Siedlung bei Hul, Slowakei. Unpublizierte MSc-Arbeit. Universität Heidelberg. Heidelberg 2016.

Lange/Knapp/Jeschke 1986 - E. Lange/H. D. Knapp/L. Jeschke: Die Landschaftsgeschichte der Insel Rügen seit dem Spätglazial. Schriften zur Ur- und Frühgeschichte $38=$ Ralswiek und Rügen. Landschaftsentwicklung und Siedlungsgeschichte der Ostseeinsel 1. Berlin 1986.

Müller-Scheeßel u. a. 2016 - N. Müller-Scheeßel/J. Bátora/ S. Reiter/K. Radloff/P. Tóth: Prospection Results in the Žitava Valley. Študijné zvesti AÚ SAV 60, 2016, 79-96.

Müller-Scheeßel u. a. 2020 - N. Müller-Scheeßel/J. Bátora/ J. Gresky/S. S. Reiter/K. Stucky/K. Rassmann: In search of the modus operandi. Reopenings of Early Bronze Age burials at Fidvár near Vráble, south-west Slovakia. In: E. Aspöck/A. Klevnäs/N. Müller-Scheeßel (eds.): Grave disturbances: The archaeology of post-depositional interactions with the dead. Studies in Funerary archaeology 14. Oxford 2020, 189-205.

Nowaczinski u. a. 2015 - E. Nowaczinski/G. Schukraft/ C. Keller/S. Hecht/B. Eitel/O. Bubenzer: Fluvial dynamics of the Žitava River, SW Slovakia, during the last 45 $\mathrm{ka} B \mathrm{P}$ and their influence on Early Bronze Age human occupation. Quaternary International 370, 2015, 113-126.

Pauly 1932 - A. F. Pauly: s. v. Synoikismos. In: Paulys Real-Encyclopädie der classischen Altertumswissenschaft. R. 2, Bd. 8. Stuttgart 1932, 1435-1446.

Rassmann 2016 - K. Rassmann: Territorien, Kontrolle und Konflikte. Befestigte Siedlungen als Spiegel sozialer Veränderungen in der Bronzezeit. In: W.-R. Busch (Hrsg.): Vor 3000 Jahren - Die erste Geesthachterin und ihre Zeit. Annäherungen an den Fund einer Frau aus der älteren Bronzezeit von Grünhof-Tesperhude, Ortsteil von Geesthacht. Geesthacht 2016, 153-167.

Rassmann u. a. 2017 - K. Rassmann/S. S. Reiter/ J.Bátora/N. Müller-Scheeßel: The Vráble toolbox: A multidisciplinary investigation of settlement change. In: B. S. Heeb/A. Szentmiklosi/R. Krause/M. Wemhoff (eds.): Fortifications: the rise and fall of defended sites in Late Bronze and Early Iron Age of South-East Europe. International conference in Timişoara, Romania from November $11^{\text {th }}$ to $13^{\text {th }}, 2015$. Berliner Beiträge zur Vorund Frühgeschichte 21. Berlin 2017, 79-90. 
Samuel 1995 - M. Samuel: Praveké a stredoveké nálezy z Hulu. AVANS 1993, 1995, 117, 118.

Sherratt 1983 - A. Sherratt: The Development of Neolithic and Copper Age Settlement in the Great Hungarian Plain Part II: Site Survey and Settlement Dynamics. Oxford Journal of Archaeology 2, 1983, 13-41.

Skorna/Bátora/Kalmbach 2018 - H. Skorna/J. Bátora/ J. Kalmbach: Vráble, Slowakei. Herausbildung und Niedergang des frühbronzezeitlichen Siedlungszentrums - Untersuchungen zu Wirtschaft, Sozialstruktur und politischer Organisation eines Sozialverbandes und seines Umfeldes. Überblick und die
Arbeiten in der Siedlung 2017. e-Forschungsberichte des DAI, 2018, 101-108.

Stucky 2018 - K. Stucky: Vráble, Slowakei. Die Forschungen auf dem frühbronzezeitlichen Gräberfeld. Die Arbeiten seit 2015. e-Forschungsberichte des DAI, 2018, 96-100.

Točík 1981 - A. Točík: Prieskumy a záchranné výskumy na juhozápadnom Slovensku v roku 1980. AVANS 1980, 1981, 296-311.

Točík 1986 - A. Točík: Opevnené sídlisko zo staršej doby bronzovej vo Vrábloch. Slovenská archeológia 34, 1986, 463-476.

Manuskript angenommen am 30. 7. 2020

Abstract translated by Nils Müller-Scheeßel

Súhrn preložila Anitra Kozubová

Dr. Nils Müller-Scheeßel

Institut für Ur- und Frühgeschichte

Christian-Albrechts-Universität zu Kiel

Johanna-Mestorf-Straße 2-6

D - 24118 Kiel

nils.mueller-scheessel@ufg.uni-kiel.de

Dr. Knut Rassmann

Römisch-Germanische Kommission des Deutschen Archäologischen Instituts

Palmengartenstraße 10-12

D - 60325 Frankfurt a. M.

knut.rassmann@dainst.de

Philipp Gerbecks, M. sc.

Goethestraße 30

D - 53113 Bonn

p.gerbecks@googlemail.com

\title{
Opevnenia zo staršej doby bronzovej v hornom Požitaví: procesy synoikizmu a dioikizmu
}

\author{
Nils Müller-Scheeßel - Philipp Gerbecks - Knut Rassmann
}

\author{
SÚHRN
}

Stav archeologického terénneho bádania na juhozápadnom Slovensku je vynikajúci a uvedené konštatovanie platí najmä pre oblast' Požitavia. Predtým neznáme miesta boli objavené dial'kovým prieskumom alebo geofyzikálnou prospekciou. Medzi ne patria aj opevnenia zo staršej doby bronzovej v Hule a Vlkase. Ich celková plocha zaberá okolo 0,5 ha a pravdepodobne išlo o sídliská pre relatívne malé sociálne skupiny. Výnimkou je v Požitaví iba lokalita Vráble-Fidvár s celkovou rozlohou až 12 ha.

Objavenie sídlisk v Hule a vo Vlkase je dôležité z viacerých dôvodov: časovo ide o lokality synchrónne so sídliskom vo Vrábloch-Fidvári, obe lokality sú rozlohou rovnako vel'ké a ich opevnenie pozostáva z priekopy s približne rovnakými rozmermi. Na rozdiel od Fidváru však sídliská v Hule (určite) a Vlkase (pravdepodobne) existovali iba krátko, možno tri až pät generácií. Nárast osídlenia vo Fidvári určite nebol spôsobený populačným rastom miestneho obyvatel'stva, ale prílivom nového obyvatel'stva zvonku. Preto predpokladáme, že v priebehu procesu synoikizmu boli ostatné miesta dobrovolne alebo nedobrovolne opustené a ich sociálne skupiny sa pravdepodobne prestahovali do Vráblov. Zároveň môžeme predpokladat', že uvedený proces viedol aj k sociálnemu členeniu a hierarchizácii, v rámci ktorej mala dominantné postavenie malá skupina z pôvodného osídlenia. Tento stav netrval viac ako 200 rokov a na konci staršej doby bronzovej v priebehu procesu dioikizmu bolo sídlisko vo Vrábloch-Fidvári opustené. V osídlení Požitavia potom opät dominovali rozlohou malé sídliská. 
